www.nature.com/pj

\title{
Electrospun poly(vinyl alcohol) nanofibers: effects of degree of hydrolysis and enhanced water stability
}

\author{
Jong-Chul Park ${ }^{1}$, Takeru Ito ${ }^{1}$, Kyu-Oh Kim ${ }^{1}$, Kwan-Woo Kim ${ }^{1}$, Byoung-Suhk Kim ${ }^{1}$, Myung-Seob Khil ${ }^{2}$, \\ Hak-Yong $\mathrm{Kim}^{2}$ and Ick-Soo Kim ${ }^{1}$
}

Polymer Journal (2010) 42, 273-276; doi:10.1038/pj.2009.340; published online 20 January 2010

Keywords: degree of hydrolysis; electrospinning; poly(vinyl alcohol); solution properties; water resistance

\section{INTRODUCTION}

Poly(vinyl alcohol) (PVA) is a highly hydrophilic, nontoxic and biocompatible semicrystalline polymer with excellent properties such as strength, water solubility, gas permeability and thermal characteristics. PVA is available in a variety of degrees of hydrolysis (DH) because it is derived from the hydrolysis (or alcoholysis) of poly(vinyl acetate) (PVAc). ${ }^{1-3}$ The properties of PVA are therefore affected by DH. A PVA with DH between 87 and $89 \%$ has lower mechanical and water resistance than a PVA with DH between 98 and $99.9 \%$. Consequently, the potential to interact with other polar polymers would be expected to vary as a function of DH. In recent years, electrospinning has become of great interest, not only because it can produce polymer fibers with diameters in the range of nano- to a few micrometers using polymer solutions or melts but also because it has the advantage of being a simple, conventional method with which a wide range of porous structures can be produced, as well as being inexpensive as compared with conventional methods. ${ }^{4-8}$ The electrospinning of the PVA solution has been extensively studied for the preparation of ultrafine separation filters, biodegradable mats and inorganic fibers. The dissolution of PVA depends on the nature of the solvent, the temperature and the extent of hydrolysis in the polymer. Depending on the rheological characteristics of the solution, electrospinning of a polymer solution can produce a variety of structures, mostly including beads, beaded fibers and fibers. ${ }^{9,10}$ Various solution parameters affecting the morphology and diameter of electrospun PVA fibers, such as the solution concentration, molecular weight, ${ }^{11}$ $\mathrm{pH},{ }^{12}$ salt, ${ }^{13}$ surfactant molecules ${ }^{14}$ and so on, have been investigated. However, the effect of DH on the morphology and diameter of electrospun PVA fibers has been less studied. In addition, PVA forms excellent nanofibers through electrospinning, but its applications are limited by its high hydrophilicity, through which it dissolves immediately on contact with water. Therefore, PVA fibers have been modified by either chemical ${ }^{15}$ or physical ${ }^{16}$ crosslinking to improve their mechanical properties and water resistance. In this study, we report the effects of $\mathrm{DH}$ on the morphology and diameter of electrospun PVA fibers and also explore the water resistance of electrospun PVA/poly(acrylic acid) (PAA) composite fibers through heat treatment.

\section{EXPERIMENTAL PROCEDURE}

\section{Materials}

PVA with various degrees of hydrolysis ( $\mathrm{DH}=88,96$ and $99.9 \%$ hydrolyzed) and the same degree of polymerization $=1700$ was kindly provided by Kuraray Co. Ltd, Tokyo, Japan. The PVA solution with a DH of $92 \%$ was separately prepared by mixing the same weight ratio $(1: 1, \mathrm{v} / \mathrm{v})$ of two PVA solutions with a DH of 88 and $96 \%$. PAA with a molecular weight of $250 \mathrm{kDa}$ was purchased from Wako, Osaka, Japan. All chemicals were of analytical grade and were used without further purification. Distilled water was used as the solvent.

\section{Electrospinning}

A high-voltage power supply (Har-100*12, Matsusada Co., Tokyo, Japan), capable of generating voltages up to $100 \mathrm{kV}$, was used as the source of the electric field. The PVA solutions dissolved in distilled water were supplied through a plastic syringe attached to a capillary tip with an inner diameter of $0.6 \mathrm{~mm}$. The copper wire connected to a positive electrode (anode) was inserted into the polymer solution, and a negative electrode (cathode) was attached to a metallic collector. The voltage was fixed at $10 \mathrm{kV}$. The distance between the capillary tip and the collector was fixed to be $15 \mathrm{~cm}$, and the plastic syringe was placed at an angle of $10^{\circ}$ from the horizontal direction. The concentration of PVA solutions was in the range of $8-12 \mathrm{wt} \%$. To improve the solubility of the PVA polymer, the PVA solution was heated to about $80^{\circ} \mathrm{C}$ so as to disrupt the strong intra- and interchain bonding that may exist in PVA polymers. PVA/PAA composite fibers were obtained using the same strategy. Briefly, PVA/PAA solutions for electrospinning were prepared by blending the PVA solution with the PAA solution at various PAA weight fractions $\left(\varphi_{\text {PAA }}\right)$. The concentrations of both the PVA and the PAA solutions were controlled to be ca. $12.0 \mathrm{wt} \%$. All solutions were electrospun onto a rotating metallic collector at room temperature under identical conditions.

\footnotetext{
${ }^{1}$ Nano Fusion Technology Research Lab., Faculty of Textile Science and Technology, Shinshu University, Nagano, Japan and ${ }^{2}$ Department of Textile Engineering, Chonbuk National University, Jeonju, South Korea

Correspondence: Dr B-S Kim or Professor I-S Kim, Nano Fusion Technology Research Lab., Faculty of Textile Science and Technology, Shinshu University, 3-15-1 Tokida, Ueda, Nagano 386-8567, Japan.

E-mail: bskim@shinshu-u.ac.jp or kim@shinshu-u.ac.jp

Received 11 September 2009; revised 11 November 2009; accepted 6 December 2009; published online 20 January 2010
} 


\section{Measurement}

The conductivity and surface tension of PVA solutions were measured using a conductivity meter (G-series, CM-40G, TOA Electronics Ltd, Aichi, Japan) and a Wilhelmy plate method with a tensiometer (CBVP-Z, Kyowa Interface Science Co. Ltd, Saitama, Japan), respectively. Viscosities were measured using a viscometer (DV-1, Brookfield Co., Stoughton, MA, USA) with spindle No. 63 at 100 r.p.m. at room temperature. Fiber morphology, average fiber diameter and distribution of the electrospun PVA fibers were characterized using scanning electron microscopy (SEM, VE-8800, Keyence Co., Tokyo, Japan) on samples sputtered with Palladium-Platinum.

\section{RESULTS AND DISCUSSION}

Electrospinning occurs when the applied electrical voltage exceeds a critical electrical potential at which the electrostatic force overcomes the surface tension of the polymer solution. Therefore, a low surface tension is desirable in electrospinning, as it reduces the critical voltage $\left(V_{\mathrm{c}}\right)$ needed for the ejection of the jet from the Taylor's cone. The surface tension of aqueous PVA solutions exhibits a marked dependence on the DH of the PVA, which may result in an altered spinnability. For instance, Wnek and colleagues ${ }^{14}$ reported that the addition of small amounts of Triton surfactant improves the electrospinnability of fully ( $>99 \%$ ) hydrolyzed PVA, probably because of lowered surface tension and retarded PVA gelation, and therefore affords reproducible electrospinning. Figure 1 (top) shows SEM images of PVA fibers electrospun from the solution with various $\mathrm{DH}$. The concentration of the PVA solution was $8.0 \mathrm{wt} \%$, which is the
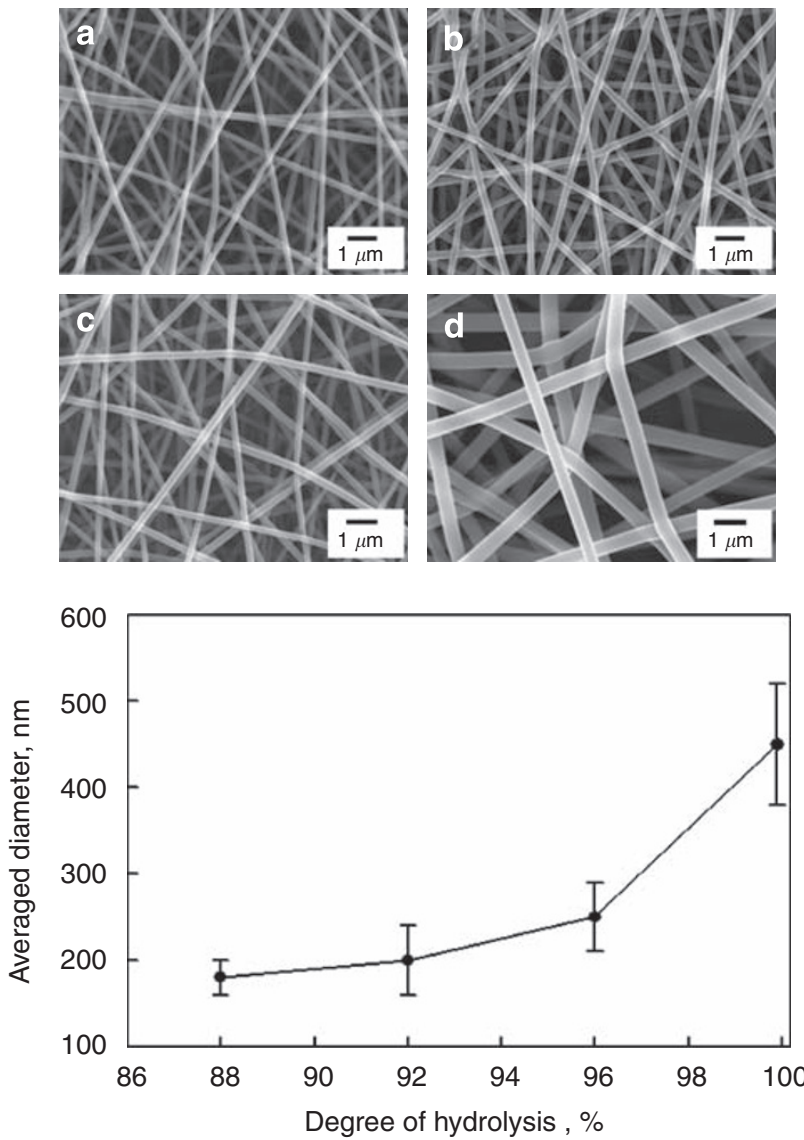

Figure 1 SEM images of electrospun PVA fibers with DH of (a) $88 \%$, (b) $92 \%$, (c) $96 \%$ and (d) $99.9 \%$ (top), and fiber diameters of the corresponding electrospun PVA fibers (bottom). The concentration of the PVA solution was $8.0 \mathrm{wt} \%$. minimum polymer concentration for fiber formation in our study. Below this critical value, the application of voltage resulted in electrospraying or bead formation, primarily because of a Rayleigh instability. At a given concentration, the fiber diameter and interfiber spacing increased with increasing DH of the PVA. The average diameters of the PVA fibers electrospun from the solutions with different DH of $88,92,96$ and $99.9 \%$ were determined to be 190 , 200, 220 and $470 \mathrm{~nm}$, respectively. The fiber diameter increased exponentially with respect to the DH of the PVA (Figure 1, bottom). In addition, in the case of $\mathrm{DH}=99.9 \%$, poor spinnability was observed because of a combination of higher surface tension and the tendency of PVA to undergo gelation through a strong hydrogen bonding.

To investigate the effects of DH on the spinnability of PVA, PVA solutions with various $\mathrm{DH}$ were prepared, and solution properties, such as viscosity, electrical conductivity and surface tension, were studied. Figure 2 shows changes in (a) viscosity and (b) surface tension of PVA solutions with various $\mathrm{DH}$ as a function of solution concentration. As seen in Figure 2a, viscosity rapidly increased at a PVA concentration of $10 \mathrm{wt} \%$, and this is responsible for the topological restrictions on molecular motion resulting from the fact that the chains cannot pass through each other, as well as being strongly dependent on the DH of the PVA. In aqueous solutions, it can be considered that the inter- and intramolecular interactions between the polar hydroxyl groups in the PVA molecules occur through hydrogen bonding and therefore influence the rheological properties
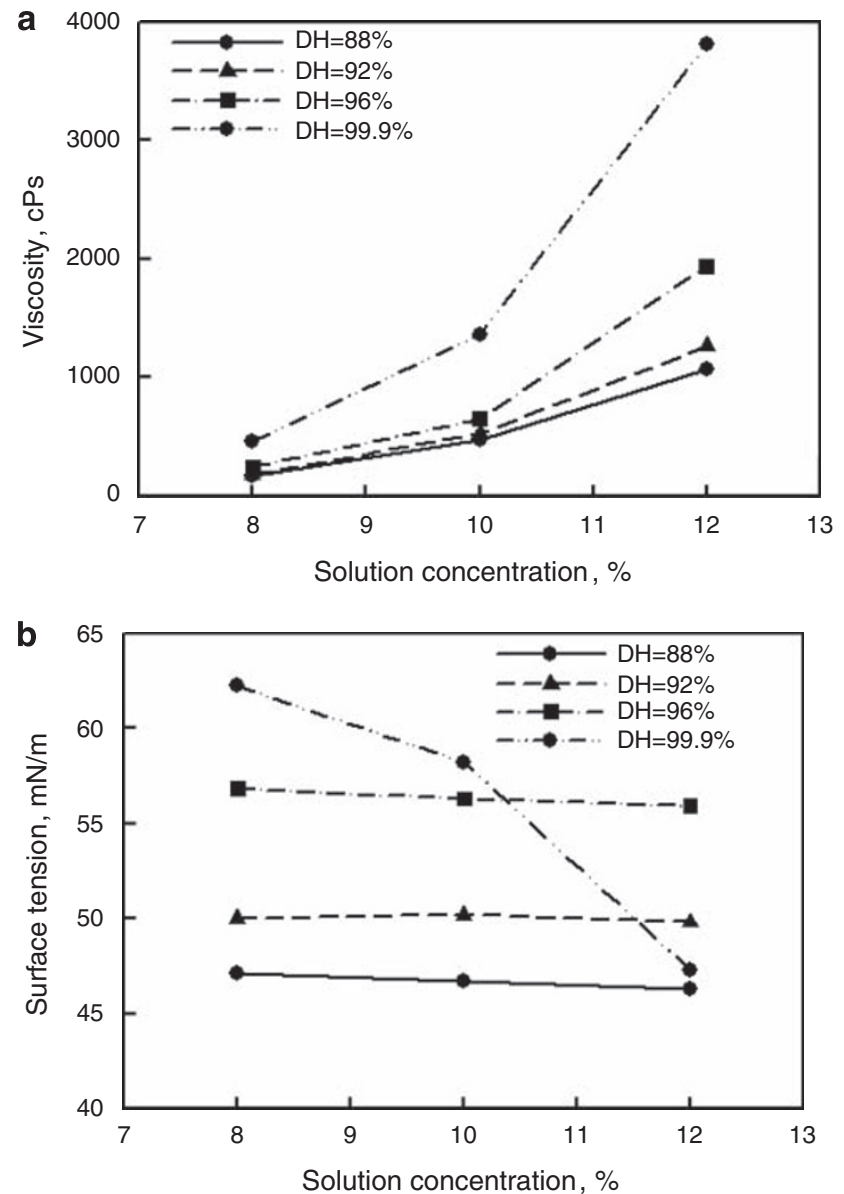

Figure 2 Changes in the (a) viscosity and (b) surface tension of PVA solutions with different $\mathrm{DH}$ as a function of solution concentration at room temperature. 

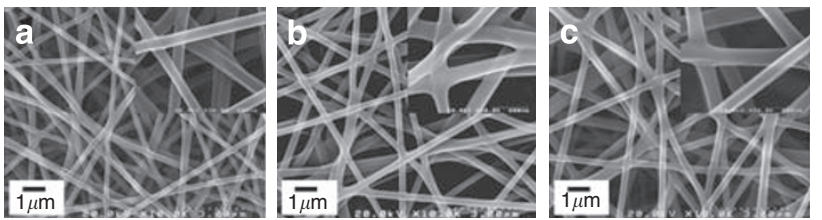

Figure 3 SEM images of PVA/PAA composite fibers electrospun from solutions with various $\varphi_{\text {PAA }}$. The concentration of both PVA and the PAA solutions was $8.0 \mathrm{wt} \%$. (a) $\varphi_{\mathrm{PAA}}=0.25$, (b) $\varphi_{\mathrm{PAA}}=0.50$ and (c) $\varphi_{\mathrm{PAA}}=0.75$.

of the PVA solutions. Moreover, the surface tension of PVA solutions at a constant concentration markedly increased with increasing $\mathrm{DH}$ of the PVA, whereas the surface tension was not affected by the solution concentration of the PVA (Figure 2b). Specifically, in the case of $\mathrm{DH}=99.9 \%$, the decreased surface tension at higher concentration may be due to the formation of strong intra- and interchain bonding in PVA chains, giving rise to physically gelling structures. ${ }^{11}$ As expected, the average diameter of the electrospun PVA fibers $(\mathrm{DH}=88 \%)$ increased with increasing solution concentration of the PVA, and this value was found to be 190,280 and $350 \mathrm{~nm}$ for the solution concentrations of 8,10 and $12 \mathrm{wt} \%$, respectively.

In addition, PVA/PAA composite fibers were prepared by the electrospinning of PVA/PAA blend solutions with different PAA weight fractions $\left(\varphi_{\text {PAA }}\right)$. Compared with electrospun pure PVA fibers, electrospinnability was markedly improved, probably because of a dramatically decreased surface tension, which is discussed later. Figure 3 shows SEM images of PVA/PAA composite fibers electrospun from solutions with various $\varphi_{\text {PAA }}$. The concentration of both the PVA and PAA solutions was fixed at $8.0 \mathrm{wt} \%$. As seen in Figure 3, the resultant PVA/PAA composite fibers were rather uniform, and the fiber diameter and its distribution increased with increasing $\varphi_{\text {PAA }}$ in the PVA/PAA blend solutions. However, in the case of pure PAA, many beads were observed, and the distribution of the fiber diameter was much broader (data not shown). By contrast, the PVA/PAA blends exhibited better electrospinnability than did pure PAA. The average diameters of the PVA/PAA composite fibers electrospun from the blend solutions with different $\varphi_{\text {PAA }}$ values of $0.25,0.50$ and 0.75 were determined to be $300 \pm 80,290 \pm 90$ and $220 \pm 50 \mathrm{~nm}$, respectively. Figure 4 shows the viscosity of the PVA/PAA blend solutions as a function of $\varphi_{\text {PAA }}$. As seen in Figure 4 , viscosity initially increased dramatically, then reached a maximum at $\varphi_{\mathrm{PAA}}=0.50$, which is close to a molar ratio of $[-\mathrm{OH}$ in $\mathrm{PVA}] /[-\mathrm{COOH}$ in $\mathrm{PAA}] \cong 1.0$ (here, $\varphi_{\text {PAA }} \approx 0.60$ ), and finally decreased. As a result, the change in solution viscosity can be considered as the $-\mathrm{OH}$ groups in PVA forming an intermolecular complex with the $-\mathrm{COOH}$ groups in PAA through hydrogen bonding. It may therefore be expected that the strongest interactions between PVA and PAA occur at around $\varphi_{\mathrm{PAA}}=0.5-0.6$, and thereby bring about the highest viscosity.

Not surprisingly, as-spun pure PVA fibers are readily soluble in water at room temperature. Therefore, to improve the water resistance of electrospun PVA fibers, PVA/PAA blend solutions with different $\varphi_{\text {PAA }}$ values were electrospun, and the resulting PVA/PAA composite fibers were heat treated at $100{ }^{\circ} \mathrm{C}$ for $1 \mathrm{~h}$. Figure 5 shows SEM images of PVA/PAA composite fibers after contact with pure water for $1 \mathrm{~h}$, followed by vacuum drying. As seen in Figure 5, interestingly, for the PVA/PAA composite fibers with $\varphi_{\mathrm{PAA}}=0.5$, the fibrous morphologies were completely conserved, indicating a dramatically enhanced water resistance of the corresponding PVA/PAA composite fibers. ${ }^{17}$ This indicates that chemical crosslinking between PVA and PAA that occurred through an ester formation between the $-\mathrm{OH}$ groups in
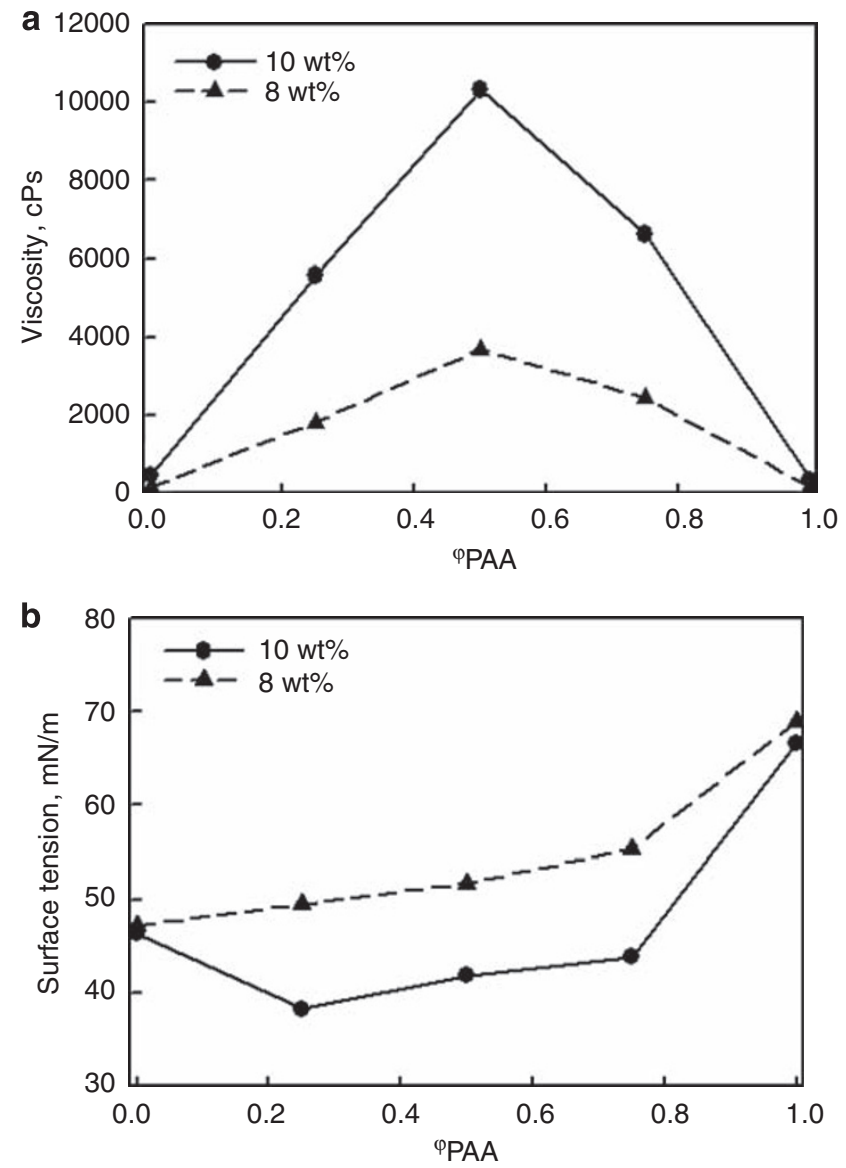

Figure 4 Changes in the (a) viscosity and (b) surface tension of PVA/PAA blend solutions as a function of $\varphi_{\text {PAA }}$. The arrow indicates the $\varphi_{\text {PAA }}$ value for the formation of stoichiometric hydrogen bonding between the $-\mathrm{OH}$ in PVA and the $-\mathrm{COOH}$ in PAA.
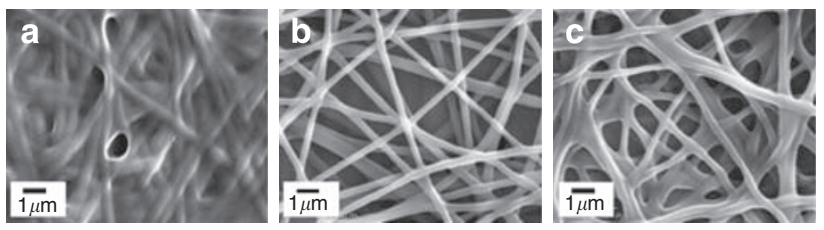

Figure 5 SEM images of PVA/PAA composite fibers after contact with water for $1 \mathrm{~h}$, followed by vacuum drying. (a) $\varphi_{\mathrm{PAA}}=0.25$, (b) $\varphi_{\mathrm{PAA}}=0.50$ and (c) $\varphi_{\mathrm{PAA}}=0.75$.

PVA and the $-\mathrm{COOH}$ groups in PAA during heat treatment at $100^{\circ} \mathrm{C}$ for $1 \mathrm{~h}$, which was confirmed by the Fourier transform infrared (FT-IR) analysis (Figure 6). Even though the FT-IR spectrum of heat-treated PVA/PAA composite fibers $\left(\varphi_{\mathrm{PAA}}=0.75\right.$, Figure $\left.6 \mathrm{~b}\right)$ shows a considerable amount of $-\mathrm{OH}$ groups $\left(3450 \mathrm{~cm}^{-1}\right)$ left in the polymers, it can be clearly seen that the $-\mathrm{C}=\mathrm{O}$ stretching vibration at $1640 \mathrm{~cm}^{-1}$ becomes stronger after heat treatment. This indicates that the $-\mathrm{C}=\mathrm{O}$ stretching vibration $\left(1715 \mathrm{~cm}^{-1}\right)$ is normally shifted to the right (lower frequencies) through an ester $(-(\mathrm{C}=\mathrm{O}) \mathrm{O})$ formation between the $-\mathrm{OH}$ groups in PVA and the $-\mathrm{COOH}$ groups in PAA. Similar results were also observed for other samples.

The water stability of the heat-treated PVA/PAA composite fibers was also affected by $\varphi_{\text {PAA }}$ in the PVA/PAA blend solutions. In the case of PVA/PAA composite fibers with $\varphi_{\mathrm{PAA}}=0.25$ and 0.75 , the typical porous structures and fiber morphologies in the electrospun nanofiber 


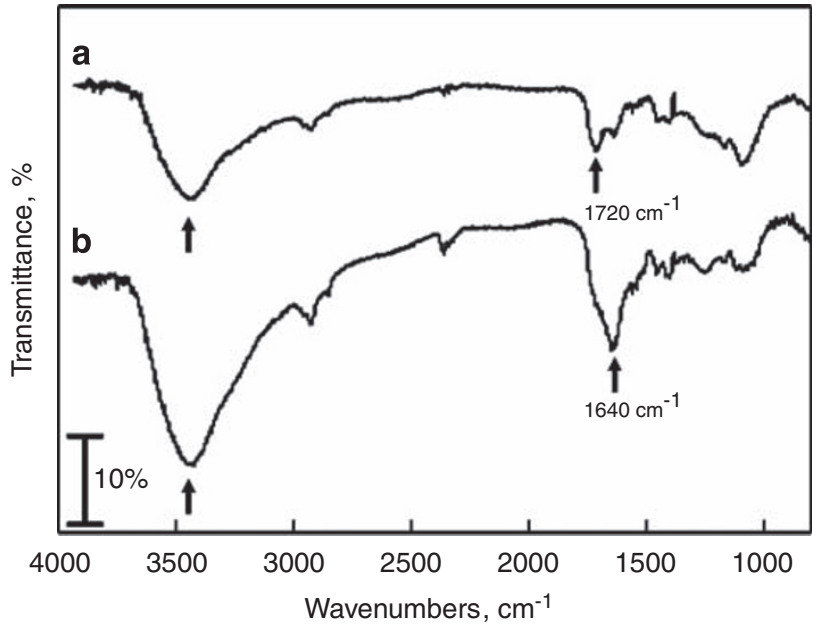

Figure 6 Fourier transform infrared spectra of PVA/PAA composite fibers $\left(\varphi_{\mathrm{PAA}}=0.75\right)(\mathbf{a})$ before and $(\mathbf{b})$ after heat treatment at $100^{\circ} \mathrm{C}$ for $1 \mathrm{~h}$.

disappeared because of partial swelling of excess amounts of either PVA or PAA on contact with water, suggesting that the degree of crosslinking was smaller than that of PVA/PAA composite fibers with $\varphi_{\mathrm{PAA}}=0.5$. It was concluded that water resistance increased by heat treatment and also depended on $\varphi_{\text {PAA }}$. Here, it should also be noted that extensive heat treatment at longer times and higher temperatures can also result in degradation of the resultant PVA/PAA composite nanofibers.

\section{CONCLUSION}

In summary, we examined the effects of the DH of PVA on electrospinning and also explored an enhanced water resistance of electrospun PVA/PAA composite fibers through heat treatment. We found that, at a constant concentration of PVA solution ( $\sim 8 \mathrm{wt} \%)$, the average diameter of the electrospun PVA fibers increased with increasing $\mathrm{DH}$, and these values were found to be $\sim 190,200,220$ and $470 \mathrm{~nm}$ for the PVA solutions of DH of 88, 92, 96 and $99.9 \%$, respectively. Moreover, the solution viscosity of PVA in water rapidly increased from $10 \mathrm{wt} \%$ of the PVA concentration and strongly depended on the DH of the PVA. In addition, the water resistance of heat-treated PVA/PAA composite fibers was dramatically enhanced and was also affected by $\varphi_{\text {PAA }}$ in PVA/PAA blend solutions.

\section{ACKNOWLEDGEMENTS}

This work was supported by a project of the 'Innovation Creative Center for Advanced Interdisciplinary Research Area' in the Special Coordination Funds for Promoting Science and Technology from the Ministry of Education, Culture, Sports, Science and Technology of Japan. BSK acknowledges the support of Grant-in-Aid for the Global COE Program by the Ministry of Education, Culture, Sports, Science and Technology, Government of Japan.

1 Finch, C. A. in: Poly(vinyl alcohol) Development (ed. Finch, C. A.) Ch. 1, 1-29 (John Wiley \& Sons Ltd, Chichester, UK, 1992).

2 Chiellini, E., Corti, A., D'Antone, S. \& Solaro, R. Biodegradation of poly(vinyl alcohol) based materials. Prog. Polym. Sci. 28, 963-1014 (2003).

3 DeMerlis, C. C. \& Schoneker, D. R. Review of the oral toxicity of polyvinyl alcohol (PVA). Food Chem. Toxicol. 41, 319-326 (2003).

4 Tan, S. H., Inai, R., Kotaki, M. \& Ramakrishna, S. Systematic parameter study for ultra-fine fiber fabrication via electrospinning process. Polymer 46, 6128-6134 (2005).

5 Lee, K. H., Ohsawa, O., Lee, S., Park, J. C., Kim, K. W., Kim, H. Y., Watanabe, Y. \& Kim, I. S. A study on the PVAc/FeSO 4 composite nanofiber via electrospinning. Sen'i Gakkaishi 64, 306-311 (2008).

6 Park, J. H., Kim, B.S., Yoo, Y. C., Khil, M. S. \& Kim, H. Y. Enhanced mechanical properties of multilayer nano-coated electrospun nylon 6 fibers via a layer-by-layer selfassembly. J. Appl. Polym. Sci. 107, 2211-2216 (2007).

7 Doshi, J. \& Reneker, D. H. Electrospinning process and applications of electrospun fibers. J. Electrostat. 35, 151-160 (1995).

8 Wei, K., Ohta, T., Kim, B. S., Lee, K. H., Khil, M. S., Kim, H. Y. \& Kim, I. S. Development of electrospun metallic hybrid nanofibers via metallization. Polym. Adv. Technol. doi:10.1002/pat.1490 (in press).

9 Shenoy, S. L., Bates, W. D., Frisch, H. L. \& Wnek, G. E. Role of chain entanglements on fiber formation during electrospinning of polymer solutions: good solvent, non-specific polymer-polymer interaction limit. Polymer 46, 3372-3384 (2005).

10 McKee, M. G., Wilkes, G. L., Colby, R. H. \& Long, T. E. Correlations of solution rheology with electrospun fiber formation of linear and branched polyesters. Macromolecules 37, 1760-1767 (2004).

11 Koski, A., Yim, K. \& Shivkumar, S. Effect of molecular weight on fibrous PVA produced by electrospinning. Mater. Lett. 58, 493-497 (2004).

12 Son, W. K., Youk, J. H., Lee, T. S. \& Park, W. H. Effect of pH on electrospinning of poly(vinyl alcohol). Mater. Lett. 59, 1571-1575 (2005).

13 Zhang, C., Xiaoyan, Y., Wu, L., Han, Y. \& Sheng, J. Study on morphology of electrospun poly(vinyl alcohol) mats. Eur. Polym. J. 41, 423-432 (2005).

14 Yao, L., Hass, T. W., Guiseppi-Elie, A., Bowlin, G. L., Simpson, D. G. \& Wnek, G. E. Electrospinning and stabilization of fully hydrolyzed poly(vinyl alcohol) fibers. Chem. Mater. 15, 1860-1864 (2003).

15 Dai, W. S. \& Barbari, T. A. Hydrogel membranes with mesh size asymmetry based on the gradient cross-linking of poly(vinyl alcohol). J. Membr. Sci. 156, 67-79 (1999).

16 Kim, C.K., Kim, B. S., Sheikh, F. A., Lee, U. S., Khil, M. S. \& Kim, H. Y. Amphiphilic poly(vinyl alcohol) hybrids and electrospun nanofibers incorporating polyhedral oligosilsesquioxane. Macromolecules 40, 4823-4828 (2007).

17 Zeng, J., Hou, H., Wendorff, J. H. \& Greiner, A. Electrospun poly(vinyl alcohol)/poly (acrylic acid) fibers with excellent water-stability. e-Polymers no 078 1-8 (2004). 